\title{
SHORT REPORT \\ Combined use of real-time PCR and nested sequence-based typing in survey of human Legionella infection
}

\author{
T. QIN ${ }^{1,2}, H . Z_{H O U}^{1,2}, H . \mathrm{REN}^{1}$, W. SHI ${ }^{3}, \mathrm{H} . \mathrm{JIN}^{4}, \mathrm{X} . \mathrm{JIANG}^{5}$, Y. XU ${ }^{6}$, \\ M. $\mathrm{ZHOU}^{7}$, J. LI ${ }^{7}$, J. WANG ${ }^{8}, \mathrm{Z}_{\mathrm{SHAO}}{ }^{1,2}$ AND X. XU ${ }^{*} \dagger$ \\ ${ }^{1}$ State Key Laboratory for Infectious Disease Prevention and Control, National Institute for Communicable \\ Disease Control and Prevention, Chinese Center for Disease Control and Prevention, Beijing, P.R. China; \\ ${ }^{2}$ Collaborative Innovation Center for Diagnosis and Treatment of Infectious Diseases, Hangzhou, P.R. China; \\ ${ }^{3}$ Shanghai CHROMagar Microbial Technology Center, Shanghai, P.R. China; ${ }^{4}$ Shanghai Municipal Center for \\ Disease Control and Prevention, Shanghai, P.R. China; ${ }^{5}$ Huashan Hospital, Fudan University, Shanghai, P.R. \\ China; ${ }^{6}$ Baoshan Branch, Huashan Hospital, Fudan University, Shanghai, P. R. China; ${ }^{7}$ Ruijin Hospital, \\ Shanghai Jiao Tong University School of Medicine, Shanghai, P. R. China; ${ }^{8}$ Changhai Hospital, Second Military \\ Medical University, Shanghai, P.R. China
}

Received 7 October 2015; Final revision 15 December 2015; Accepted 20 December 2015; first published online 21 January 2016

\section{SUMMARY}

Legionnaires' disease (LD) is a globally distributed systemic infectious disease. The burden of LD in many regions is still unclear, especially in Asian countries including China. A survey of Legionella infection using real-time PCR and nested sequence-based typing (SBT) was performed in two hospitals in Shanghai, China. A total of 265 bronchoalveolar lavage fluid (BALF) specimens were collected from hospital A between January 2012 and December 2013, and 359 sputum specimens were collected from hospital B throughout 2012. A total of 71 specimens were positive for Legionella according to real-time PCR focusing on the 5S rRNA gene. Seventy of these specimens were identified as Legionella pneumophila as a result of real-time PCR amplification of the $\operatorname{dot} \mathrm{A}$ gene. Results of nested SBT revealed high genetic polymorphism in these L. pneumophila and ST1 was the predominant sequence type. These data revealed that the burden of LD in China is much greater than that recognized previously, and real-time PCR may be a suitable monitoring technology for LD in large sample surveys in regions lacking the economic and technical resources to perform other methods, such as urinary antigen tests and culture methods.

Key words: Bacterial infections, clinical microbiology, infectious disease, Legionella, Legionnaires' disease.

Legionnaires' disease (LD) is a form of atypical pneumonia caused by Gram-negative bacteria of the genus Legionella [1]. LD is reported less commonly in Asian countries, especially in China. In the past 10 years,

\footnotetext{
* Author for correspondence: Professor X. Xu, Shanghai Municipal Center for Disease Control and Prevention, No. 1380, Zhongshan West Road, Shanghai, 200336, People's Republic of China. (Email: xuxuebin@scdc.sh.cn)

$\dagger$ There was an error of omission in the title that has now been corrected and a notice has been published providing details.
}

cases of infection by Legionella have been reported in China only sporadically or in small outbreaks [2-5]. In China, most patients use antibiotics prior to hospital admission, and it is difficult to obtain strains from respiratory specimens. In a previous report, we described four cases of Legionella pneumophila infections associated with liver cirrhosis; of these cases three were identified by real-time polymerase chain reaction (real-time PCR) [4].

Isolation of Legionella is considered the 'gold standard' for the diagnosis, but culture-confirmed 
Table 1. Details and positive rate of respiratory specimens tested in the study

\begin{tabular}{lllllc}
\hline \hline Hospital & Period of sample collection & Sample type & No. of samples & No. of positive samples & Positive rate \\
\hline Hospital A & Jan. 2012-Oct. 2013 & BAL & 265 & 41 & $15 \cdot 47 \%$ \\
Hospital B & Jan. 2012-Dec. 2012 & Sputum & 359 & 30 & $8 \cdot 36 \%$ \\
\hline \hline
\end{tabular}

BAL, Bronchoalveolar lavage.

diagnosis of Legionella infections is often hampered by early antibiotic treatment and demanding culture requirements. The urinary antigen (UAG) test is commonly used to diagnose LD; however, the present UAG test reagents could detect only $L$. pneumophila serogroup 1 (Lp1) with non-Lp1 LD being missed. Furthermore, because of its high cost, the UAG test is unlikely to be widely used in economically underdeveloped regions. The benefit of performing PCR methods on respiratory samples is now clearly established, especially for diagnosing other species and serogroups than Lp1 [6-8]. In a study conducted by Murdoch et al. in New Zealand, a $>$ fourfold increase in the number of detected cases of LD by real-time PCR compared to culture was obtained [8]. In the present study, we undertook a retrospective survey to detect Legionella in patients with pneumonia or other lower respiratory tract infections (LRTIs). Nested sequence-based typing (SBT) was used for analyses of the population structure of real-time PCR-positive specimens.

A real-time PCR-based survey was performed in two hospitals in Shanghai, China. Real-time PCR focusing on the 5S rRNA gene, which has a specificity of $100 \%$ and a sensitivity of 10 copies of genomic DNA, as described previously, was undertaken $[4,9,10]$. Real-time reactions were conducted in a quantitative PCR instrument (Stratagene, USA) and conditions were as follows: an initial denaturing step of $95^{\circ} \mathrm{C}$ for $2 \mathrm{~min}$, followed by 40 cycles of $95^{\circ} \mathrm{C}$ for $10 \mathrm{~s}$ and $60^{\circ} \mathrm{C}$ for $20 \mathrm{~s}$. During the study period, patients were sampled who had symptoms of pneumonia or other LTRIs (cough, expectoration, moist rales on lung, and one of the following: fever, neutrophilic infiltration, or changes by chest radiography) and where Streptococcus pneumoniae and Haemophilus influenza had been excluded. A total of 265 bronchoalveolar lavage fluid (BALF) specimens were collected from hospital A between January 2012 and December 2013, and 359 sputum specimens were collected from hospital B throughout 2012. All specimens were analysed by culture method using buffered charcoal-yeast extract agar with $\alpha$-ketoglutarate (BCYE; Oxoid, UK) and no Legionella isolates were obtained from any specimen. These specimens were collected in our laboratory and tested for Legionella by real-time PCR. The overall positive rate among 624 cases was $11 \cdot 4 \%$. A total of 71 specimens were positive for Legionella according to real-time PCR: $41(15 \cdot 5 \%)$ BALF specimens from hospital A and $30(8 \cdot 4 \%)$ sputum specimens from hospital B (Table 1). Real-time PCR focusing on the $\operatorname{dot} \mathrm{A}$ gene (which is restricted in L. pneumophila) was performed to test the 71 specimens [11]. Seventy were identified as $L$. pneumophila as a result of real-time PCR amplification of the $\operatorname{dot} \mathrm{A}$ gene.

The 71 cases of Legionella infection were aged 2376 (median 58.4) years and $48(67.6 \%)$ were male. All 71 cases were community acquired as the symptoms were observed before hospital admission. Upon hospital admission, six $(8 \cdot 5 \%)$ of these cases were diagnosed with pneumonia, $43(60 \cdot 6 \%)$ had lung shadows, $17(23.9 \%)$ had fever with cough, three $(4 \cdot 2 \%)$ had pleural effusion, and two $(2 \cdot 8 \%)$ had a lung abscess. All 71 patients had changes consistent with pneumonia according to chest radiography after hospital admission. In hospital A, positive rates were slightly higher during the first and fourth quarters than in the second and third quarters, especially in 2012. In hospital B, the number of positive cases and positive rates were higher in the fourth quarter than in the other quarters (Fig. 1). There is trend of seasonal variation of positive rates and case numbers, in particular the autumn peak is consistent with the pattern found in other regions [12]; however, a survey with a larger sample size should be performed to obtain a more accurate conclusion.

All 71 real-time PCR-positive specimens were tested by nested SBT using a protocol described previously [13]. A full 7-allele profile was obtained for 44/ $71(62 \cdot 0 \%)$ specimens, a further $13 / 71(18 \cdot 3 \%)$ specimens gave 5- or 6-allele profiles [usually sufficient to identify the strain as belonging to one or two sequence types (STs)], 4/71 (5.6\%) gave 3- or 4-allele profiles (usually sufficient to differentiate one profile from another), and 9/71 (12.7\%) gave 1- or 2-allele profiles 

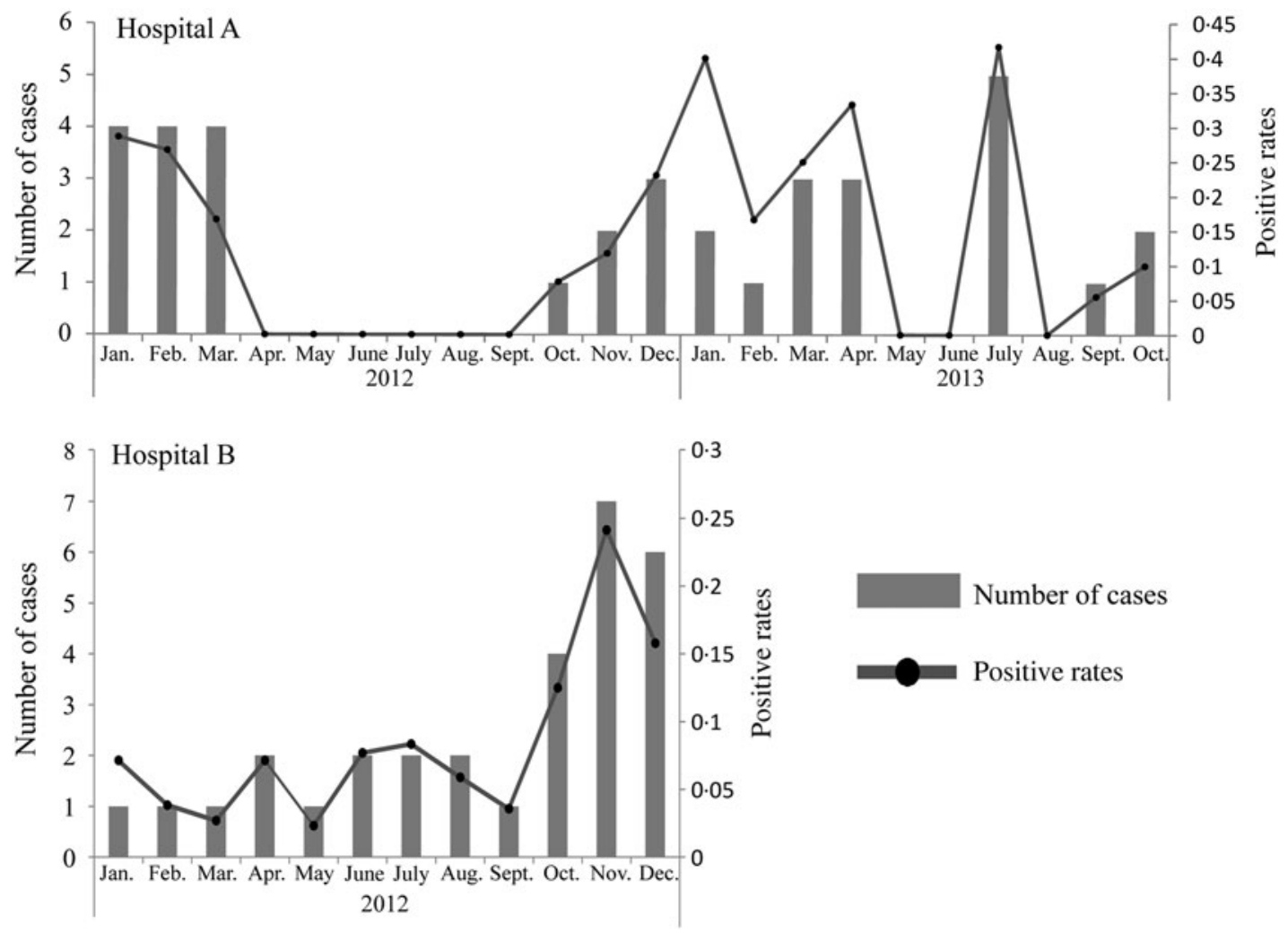

Fig. 1. Number of positive cases and positive rates of cases of Legionella infection detected by real-time PCR in two hospitals, China.

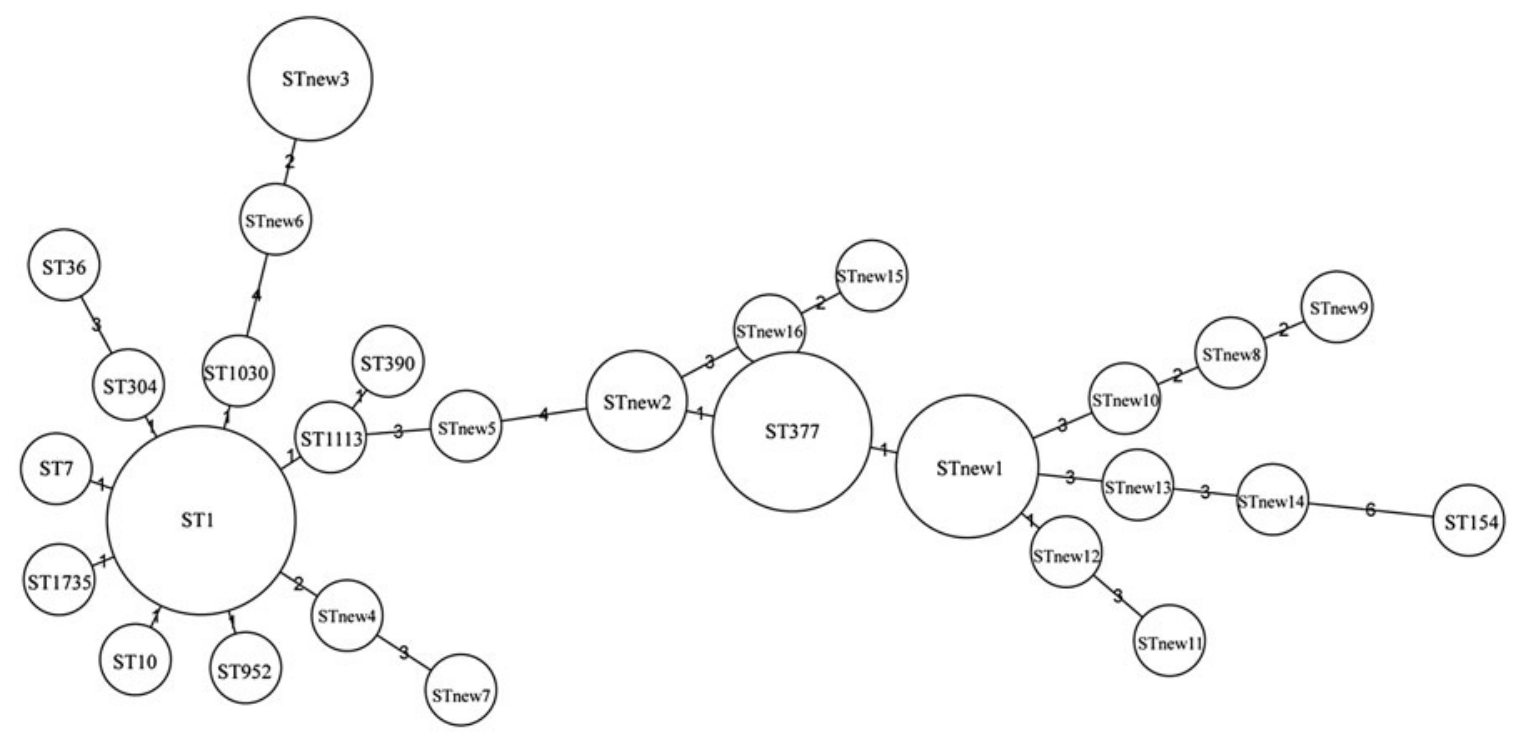

Fig. 2. Minimum spanning-tree analyses of 44 specimens with a full 7 -allele profile obtained based on sequence-based typing. Sequence types (STs) are shown as circles. The size of each circle indicates the number of isolates within this particular type. STs are shown in circles; blank circles represent new STs.

(sufficient to distinguish strains). Overall, 70/71 $(98 \cdot 6 \%)$ gave at least one allele. Of 41 BALF specimens, $80 \cdot 5 \%(n=33)$ gave a full profile. Only $36 \cdot 7 \%$ (11/30) sputum specimens gave a full profile, and
$40 \cdot 0 \%(12 / 30)$ of sputum specimens gave 5 - or 6 -allele profiles (Supplementary Table S1).

Of 44 specimens in which the full 7 -allele profile was obtained, 28 STs were identified with a 
Simpson diversity index of 0.9567 , suggesting high genetic polymorphism [14]. A minimum spanning tree based on 28 STs was structured using BioNumerics v. $7 \cdot 1$ software (www.applied-maths. com) (Fig. 2). Of the 28 STs, 12 could be assigned a ST number and the other 16 STs were identified for the first time using the EWGLI SBT database (www.ewgli.org). ST1, ST154 and ST377 were also detected in our previous environmental surveys in China $[15,16]$. Of these three STs, ST1 was the predominant ST in strains isolated from all types of water systems [16]; the ST377 strain was detected in hot springs [15]; ST154 was detected in cooling towers and hot springs [16]. This data is consistent with the widely recognized role that cooling towers and hot springs play in the causation of LD. ST1 is also the predominant ST of clinical and environmental isolates worldwide [16-18]. Seven STs (ST7, ST10, ST304, ST952, ST1030, ST1113, ST1735) in this study were single-locus variants (SLVs) of ST1. Two specimens that gave 6-allele profiles were in accordance with that of ST1. ST377 (6-10-15-289-14-1) was the second most predominant ST in this study, and was contained in five specimens.

The current system used to monitor Legionella in China is focused on environmental water samples rather than clinical samples [19, 20], and a systematic survey has not been reported. The major finding from this study is that the burden of LD in China is much greater than recognized previously. However, data regarding the UAG test is lacking and the prevalence of LD in this study is probably underestimated as several studies have shown that the UAG test can be more sensitive than PCR in respiratory samples for diagnosing LD [21]. Combining real-time PCR and the UAG test may have better application for diagnosing LD. A systemic survey synchronous with UAGs, PCR and culture should be conducted in China in the future. Furthermore, using nested SBT allows extending the results of population structure to uncultivable clinical Legionella strains.

\section{SUPPLEMENTARY MATERIAL}

For supplementary material accompanying this paper visit http://dx.doi.org/10.1017/S0950268815003301.

\section{ACKNOWLEDGEMENTS}

This study was supported by the National Natural Scientific Foundation (No. 81201251 ) from the Ministry of Science and Technology of the People's Republic of China, and the Priority Project on Infectious Disease Control and Prevention (Nos. 2013ZX10004610 and 2012ZX10004215-003) from the Ministry of Health and the Ministry of Science and Technology of the People's Republic of China.

\section{DECLARATION OF INTEREST}

None.

\section{REFERENCES}

1. Murdoch DR, Chambers ST. Atypical pneumonia-time to breathe new life into a useful term? Lancet Infectious Diseases 2009; 9: 512-519.

2. Li Y, et al. Outbreak of six cases of nosocomial Legionella pneumophila pneumonia [in Chinese]. Zhonghua Jie He He Hu Xi Za Zhi 2015; 38: 294-297.

3. Jiang L, et al. The clinical value of urinary antigen detection of Legionella pneumonia [in Chinese]. Zhonghua Jie He He Hu Xi Za Zhi 2015; 38: 29-33.

4. Qin T, et al. Liver cirrhosis as a predisposing condition for Legionnaires' disease: a report of four laboratoryconfirmed cases from China. Journal of Medical Microbiology 2012; 61: 1023-1028.

5. Zhang Q, et al. Legionnaires' disease caused by Legionella pneumophila serogroups 5 and 10, China. Emerging Infectious Diseases 2014; 20: 1242-1243.

6. Maurin M, et al. Quantitative real-time PCR tests for diagnostic and prognostic purposes in cases of legionellosis. Clinical Microbiology and Infection 2010; 16: 379-384.

7. Mentasti M, et al. Application of Legionella pneumophila-specific quantitative real-time PCR combined with direct amplification and sequence-based typing in the diagnosis and epidemiological investigation of Legionnaires' disease. European Journal of Clinical Microbiology and Infectious Diseases 2012; 31: 2017 2028.

8. Murdoch DR, et al. Impact of routine systematic polymerase chain reaction testing on case finding for Legionnaires' disease: a pre-post comparison study. Clinical Infectious Diseases 2013; 57: 1275-1281.

9. Mahbubani MH, et al. Detection of Legionella with polymerasechain reaction and gene probe methods. Molecular and Cellular Probes 1990; 4: 175-187.

10. Qin T, et al. Establishment and application of TaqMan real-time PCR in detection of Legionella spp. in environmental water samples [in Chinese]. Chinese Journal of Preventive Medicine 2011; 45: 444-447.

11. Yáñez MA, et al. Quantitative detection of Legionella pneumophila in water samples by immunomagnetic purification and real-time PCR amplification of the $\operatorname{dot} A$ 
gene. Applied and Environmental Microbiology 2005; 71: 3433-3441.

12. Bentham RH, Broadbent CR. A model for autumn outbreaks of Legionnaires' disease associated with cooling towers, linked to system operation and size. Epidemiology and Infection 1993; 111: 287-295.

13. Ginevra C, et al. Evaluation of a nested PCR-derived sequence-based typing method applied directly to respiratory samples from patients with Legionnaires' disease. Journal of Clinical Microbiology 2009; 47: 981-987.

14. Hunter PR, Gaston MA. Numerical index of the discriminatory ability of typing systems: an application of Simpson's index of diversity. Journal of Clinical Microbiology 1988; 26: 2465-2466.

15. Qin T, et al. High prevalence, genetic diversity and intracellular growth ability of Legionella in hot spring environments. PLOS ONE 2013; 8: e59018.

16. Qin T, et al. Distribution of sequence-based types of Legionella pneumophila serogroup 1 strains isolated from cooling towers, hot springs, and potable water systems in China. Applied and Environmental Microbiology 2014; 80: 2150-2157.
17. Kozak NA, et al. Distribution of lag-1 alleles and sequence-based types among Legionella pneumophila serogroup 1 clinical and environmental isolates in the United States. Journal of Clinical Microbiology 2009; 47: 2525-2535.

18. Borchardt $\mathbf{J}$, et al. Occurrence and distribution of sequence types among Legionella pneumophila strains isolated from patients in Germany: common features and differences to other regions of the world. European Journal of Clinical Microbiology and Infectious Diseases 2008; 27: 29-36.

19. Qin T, et al. Application of EMA-qPCR as a complementary tool for the detection and monitoring of Legionella in different water systems. World Journal of Microbiology and Biotechnology 2012; 28: 1881-1890.

20. Guo J, et al. Sequence types diversity of Legionella pneumophila isolates from environmental water sources in Guangzhou and Jiangmen, China. Infection, Genetics and Evolution 2015; 29: 35-41.

21. Chen DJ, et al. Utility of PCR, culture, and antigen detection for the diagnosis of legionellosis. Journal of Clinical Microbiology. Published online: 19 August 2015. doi:10.1128/JCM.01808-15. 